\title{
Design of an Open Platform for Real-Time Power Grid Monitoring
}

\author{
Qiteng Hong, Steven Blair, \\ Rhys Williams and Campbell Booth \\ Institute for Energy and Environment \\ University of Strathclyde, Glasgow, UK \\ Email: q.hong@strath.ac.uk
}

\author{
Max McFarlane \\ TNEI Services, \\ Glasgow, UK
}

\author{
Rachael Eynon \\ National Grid ESO, \\ Warwick, UK
}

\author{
Nathan Matheson \\ Siemens Gamesa Renewable \\ Energy, Glasgow, UK
}

\begin{abstract}
This paper presents the design and implementation of the UK's first open platform for acquiring, archiving, and visualizing high-resolution real-time measurement data from Phasor Measurement Units (PMUs). The platform, termed "Smart Grid Monitoring and Visualization Platform (SGMVP)", is also capable of accessing and sharing the operational data of the Great Britain (GB) transmission network (e.g. demand level, output of different generation units, power flow in interconnectors, etc.). SGMVP provides an ideal solution for open and convenient access to valuable grid data, which enables a wide range of potential applications (e.g. power system model validation, provision of training data for data-driven approaches in power systems, etc.). In this paper, the architecture of the SGMVP platform and the design and implementation of the key functional blocks will be presented in detail. Discussion of the potential use cases of the platform for various purposes is presented. Future plans for further developing the platform to incorporate more PMUs from different locations in the GB power network are discussed.

Index Terms - real-time monitoring, PMU, wide-area monitoring, protection and control, renewable generation.
\end{abstract}

\section{INTRODUCTION}

The energy landscape worldwide is experiencing significant changes with a massive increase of renewable generation. The UK, in particular, is facing significant challenges due to the rapidly growing capacity of wind and PV generation, along with the decommissioning of conventional thermal plants [1]. The increased proportion of converter-interfaced generation means the system will become more dynamic during disturbances [2]. Synchronized real-time monitoring data from Phasor Measurement Units (PMUs) is extremely valuable for the understanding of the system behavior and can contribute to a range of novel solutions for tackling renewable integration challenges [3]-[5].

Presently, there are a number of open platforms available in the UK for visualizing the grid operating status (e.g. [6], [7]), but the common shortcoming of existing platforms is they only provide grid operational data (e.g. generation output, demand, etc.) and low-resolution frequency data (typically with an update rate of $15 \mathrm{~s}$ ), which is not sufficient for many applications, e.g. power system model validation, analysis of transient and dynamic system behaviour during disturbances, etc. Outside the UK, there are a number of open platforms providing real-time virtualization of PMU data for users (e.g. [8] in the US, [9] in Switzerland, and [10] in Brazil).
However, these platforms only provide PMU-related data (e.g. frequency, angle, etc.) without comprehensive grid operational data, which also largely limits their application for research and development purposes.

A comprehensive set of grid operational data and highresolution PMU data is critical for many power system applications (detailed discussion provided in Section III). However, from the review of existing platforms, it was found that there was no open platform that offers both comprehensive PMU data and grid operational data.

Therefore, this paper presents the design and implementation of the UK's first open platform for acquisition, archiving and visualization of high-resolution synchronized data from PMUs, along with grid operational data (e.g. demand, output of different generation types, power flows in interconnectors, etc.). The SGMVP platform provides an ideal solution for open and convenient access to valuable grid data, which enables a wide range of potential applications (e.g. model validation, machine learning application in power systems, etc.).

The paper is organized as follows: Section II presents the design and implementation of the SGMVP platform; Section III discusses the potential use cases of the SGMVP platform for various power system applications; and Section IV concludes the paper with key learnings and highlights the future work.

\section{DESIGN OF THE SGMVP PlatForm}

\section{A. Overview of the SGMVP platform}

An overview of the hardware architecture that enables the SGMVP platform is illustrated in Fig. 1. The various pieces of hardware are mainly installed in two locations, i.e. the Technology and Innovation Centre (TIC) [11] and the Power Networks Demonstration Centre (PNDC) [12], which are approximately $24 \mathrm{~km}$ away from each other. The platform is hosted in a server PC in TIC, which is connected to a specifically configured Local Area Network (LAN) called "TIC-LAN". Within the same LAN, a Schweitzer Engineering Laboratories relay with PMU functionality (referred to as "TIC-PMU") was installed [13]. TIC-LAN is connected to the University LAN via a router for enhanced data flow management. A GPS clock and antenna are also installed at TIC to receive synchronized time signal for the PMU. Similar 


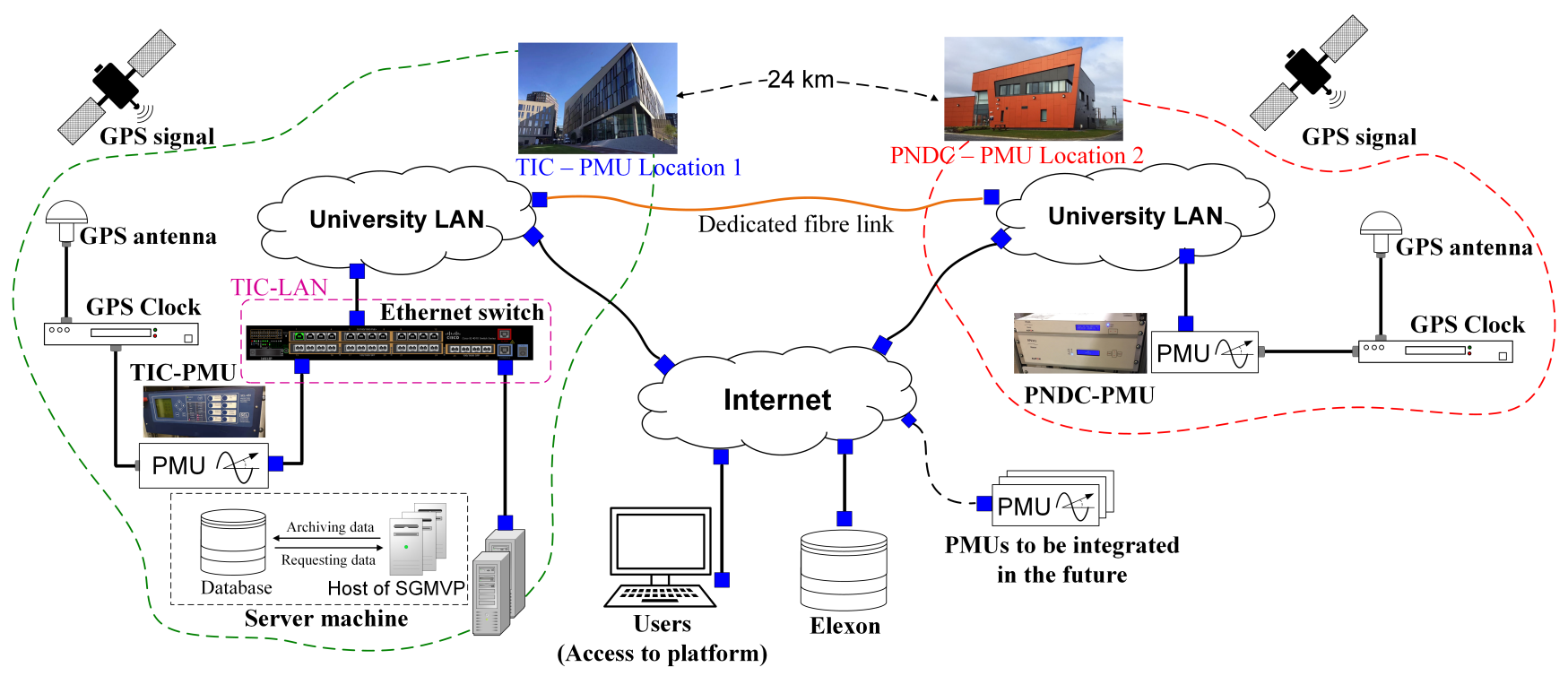

Fig. 1. Overview of the hardware architecture for supporting the SGMVP platform

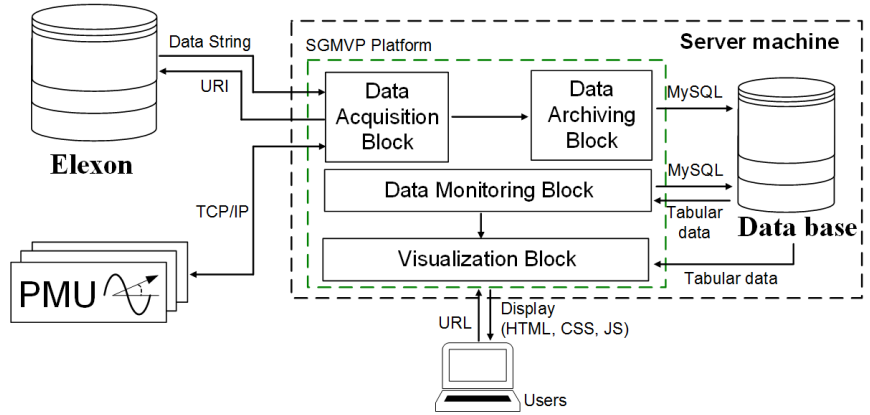

Fig. 2. Design of the SGMVP platform

arrangement is set up at PNDC for the second PMU (referred to as "PNDC-PMU") [14]. The PNDC-PMU is connected to the University LAN directly and the data is transmitted to the server via a dedicated fibre link that connects the University LANs at PNDC and TIC. The operational data is retrieved from Elexon via the internet link, which will be described in detail in section II-B. The platform is openly accessible by any users who have access to the internet [15].

\section{B. Data Acquisition}

The design of the SGMVP platform is illustrated in Fig. 2. There are two main types of data that SGMVP acquires and shares with the users, i.e. grid operational data and real-time PMU synchrophasor data. The grid operational data shows the GB electricity network status, e.g. total system demand, generation output from different types of generation units, power flows in the various HVDC interconnectors, etc. This type of data was sourced from Elexon, which offers Balancing Mechanism Reporting Service (BMRS) - a primary channel for providing the GB grid operational data to facilitate market participants in making informed trading decisions [16]. The
TABLE I

DESIGN OF THE SGMVP PLATFORM

\begin{tabular}{|l|l|l|l|}
\hline & \multicolumn{1}{|c|}{ Data } & Unit & Update rate \\
\hline \multirow{3}{*}{ Elexon } & Frequency & $\mathrm{Hz}$ & Every $15 \mathrm{~s}$ \\
\cline { 2 - 4 } & System Demand & $\mathrm{MW}$ & Every $5 \mathrm{mins}$ \\
\cline { 2 - 4 } & $\begin{array}{l}\text { Generation ouput by types } \\
\text { (e.g. wind, CCGT, nuclear, etc.) }\end{array}$ & $\mathrm{MW}$ & Every $5 \mathrm{mins}$ \\
\hline \multirow{3}{*}{ PMU } & Frequency & $\mathrm{Hz}$ & $50 \mathrm{frame} / \mathrm{s}$ \\
\cline { 2 - 4 } & RoCoF & $\mathrm{Hz} / \mathrm{s}$ & $50 \mathrm{frame} / \mathrm{s}$ \\
\cline { 2 - 4 } & Phasors (e.g. $V_{A}, V_{B}$ and $\left.V_{C}\right)$ & $\mathrm{V}$ & 50 frame/s \\
\hline
\end{tabular}

data acquired from Elexon and PMUs and their updating rates are described in Table I.

Acquiring data from the BMRS requires registering as a user of the Elexon Portal and obtaining scripting key via an Application Programming Interface (API). The API permits communication between between SGMVP and Elexon, while the key identifies the device used to request data from the portal. To access the API, a unique Uniform Resource Identifier (URI) is required for each request and detailed instructions can be found in [17].

For the PMU data acquisition, a Python script was developed to fetch the synchrophasor data from the PMU and format it for storage. The Synchrophasor library [18] was used to establish a TCP/IP connection between the PMU and server machine to transport and decode the IEEE C37.118 data packets [19].

\section{Data Archiving}

One of the most crucial elements that enables the visualization and data sharing function of the SGMVP platform is to adequately store data in a centralized location for quick and efficient access. In this work, both of the the SGMVP platform and the database are run on a physical server machine as illustrated in Fig. 2. The data archiving block contains 
and executes multiple Python scripts to manage data flow and manage efficient storage and client requests of data.

A MySQL relational database [20] was selected and set up to manage and collate structured data from multiple sources. This database stores operational data, synchrophasor data and contact details obtained when a user subscribes to system disturbance updates on the website. The database was structured to sort numerical data into tables categorized by record interval length. Operational data intervals are based on the data update rate as shown in Table I, and PMU data is posted to the database every three seconds. The interval of PMU data storage does not reflect resolution, where data packets were buffered to reduce post requests to the database.

Archiving of operational data has been designed to delay successive operations to extract the most recent data from Elexon. This was implemented under the management of a background scheduler. Python library Advanced Python Scheduler [21] was adopted to allow periodic scheduling of requests to Elexon. The request frequency for each data type was determined based on the update frequency in Table I. Each iteration completes an API request, retrieving a single string variable. This variable is then processed using a series of formatting, validation, labelling and restructuring operations to produce an SQL-syntax command. Occasional errors present throughout the operation of the data archiving process incurred a lack of consistency in data storage. This was avoided by developing a gap fill function which attempts to refill error regions with delayed request data from Elexon.

For the PMU data formatting and archiving, a limit exists on the number of write requests are made to the server within a given period to avoid overloading the server. PMU data is received at a greater rate than it can be processed at, so buffering is used to temporarily store incoming data until it can be processed by the server.

\section{Data Visualisation}

The visualization of the real-time PMU data and the near real-time operational data involves a back-end process and a front-end interface. The back-end process is for requesting and handling data from the database, while the front-end interface is mainly for displaying the data using various graphical components.

Data is continuously acquired from the database to update the live displays on the SGMVP platform. To generate dynamic page content, PHP was used to generate MySQL commands with request parameters made available through JavaScript. The original resolution of PMU data proved to overload the website visualization components. Therefore, the back-end process also introduces a mechanism to intentionally reduce the resolution of the PMU data retrieved from the database so that it is appropriate for the front-end display. The back-end process is also designed to convert the data to appropriate formats so that it is compliant to the libraries that provide the graphical components used in the front-end interface of the platform.

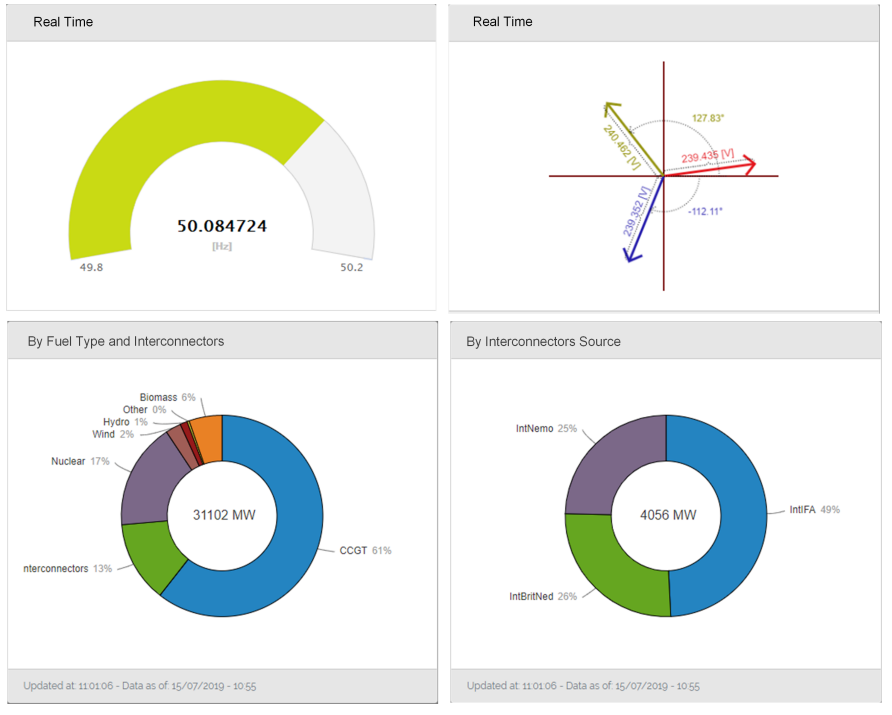

Fig. 3. Graphical gauges used in SGMVP for displaying grid status data

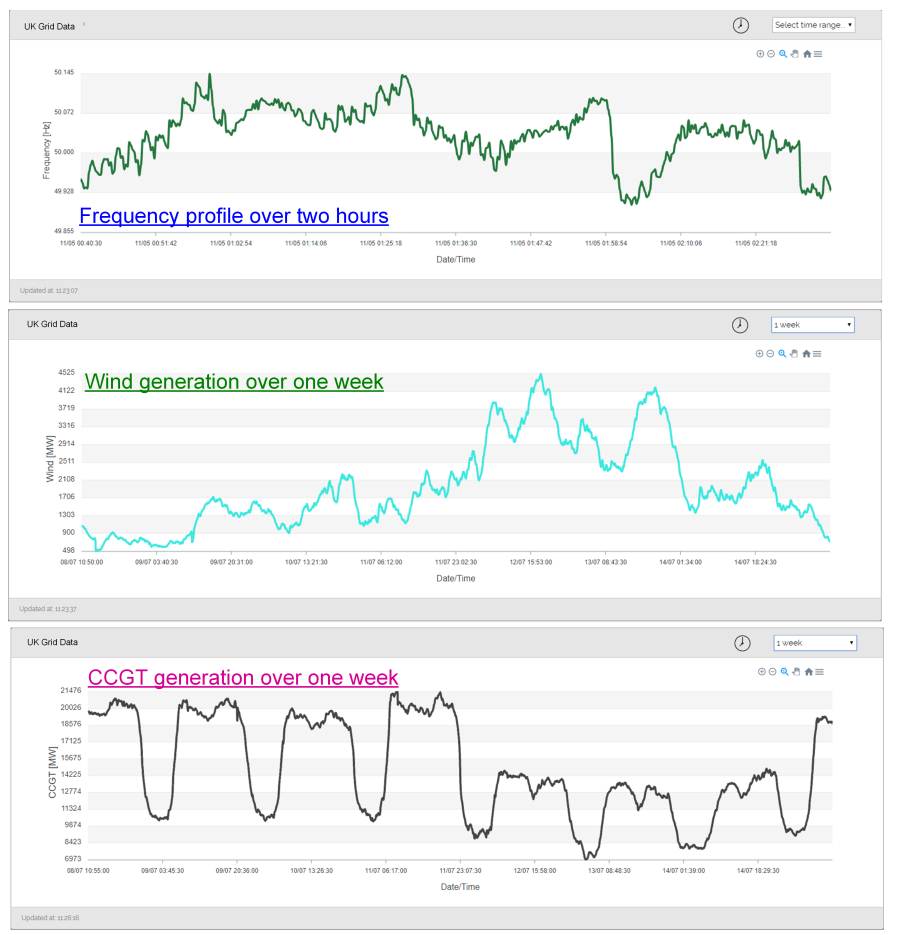

Fig. 4. Examples of historical data shown in the SGMVP platform

The front end of data visualization block is the graphical elements that users can directly see when accessing the platform. Fig. 3 shows the graphical gauges used for displaying various grid status data in the SGMVP platform, while Fig. 4 presents example curves showing historical frequency, wind and Combined Cycle Gas Turbines (CCGT) data. These graphical components were implemented using the library from [22].

The SGMVP platform is also equipped with a heat map to visualize the variation of frequency and rate-of-change-offrequency (RoCoF) measured by PMU data across different 

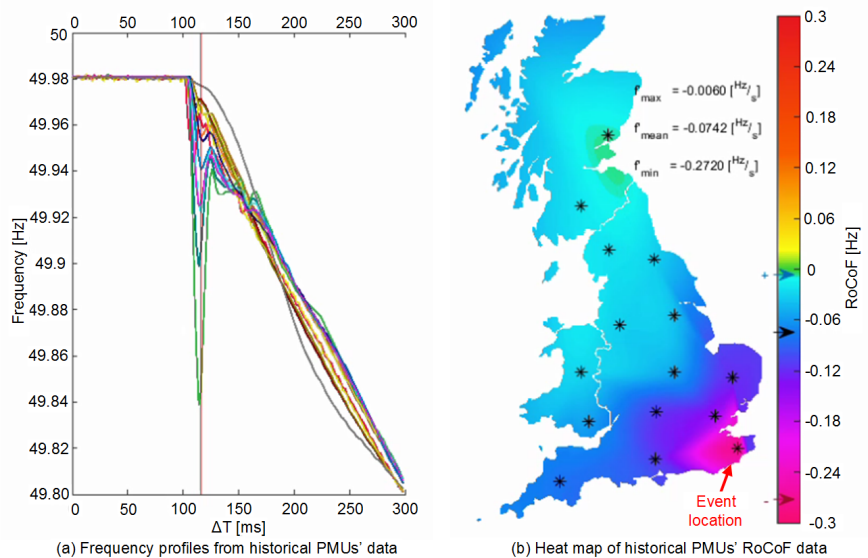

Fig. 5. Heat map for displaying historical PMU data

locations of the network. Example frequency profile and the RoCoF heat map are shown in Fig. 5. Based on the studies conducted in [2], [4], the system will experience frequency variations at different locations during power imbalance events. By analysing the characteristics of the PMU data measured at different locations, it is possible to accurately locate the event location, thus deploying the most effective control actions. As shown in the example in Fig. 5, at the location of the event, the system experiences the most severe RoCoF and through the graphical tool, this can be shown in a intuitive manner. Currently, there are only two PMUs online for providing realtime data for the SGMVP platform, so the data used for this example is from historical PMU data measured across different locations in the GB network. When there are more PMUs connected to the SGMVP platform, it is planned to enable the heat map as a live component for receiving and displaying real-time variation of frequency, RoCoF and angle information in the system at different locations.

\section{E. Monitoring of grid status}

The SGMVP is also equipped with the functions for monitoring the grid status using the measurement data from PMUs. The data monitoring block continuously monitors the frequency, RoCoF and the phasors data, where the data is compared with a number of pre-configured thresholds. If any of the measurement data is beyond the configured corresponding threshold, it is considered that there is an event in the system, and the event will be recorded with notifications sent to any users who subscribe to grid events on the platform. For example, the normal operational limit of frequency in the UK is between $49.8 \mathrm{~Hz}$ and $50.2 \mathrm{~Hz}$. If the measured frequency data is beyond this limit, it indicates there is a frequency disturbance, and the event will be recorded and notification will be sent to subscribed users.

\section{USE CASES OF THE SMART GRID MVP PLATFORM}

With the unique capability of offering both real-time high resolution PMUs data and grid operational data, the SGMVP can enable a wide range of diverse applications, which can be summarised in the following subsections.

\section{A. Research and Development}

The potential application of the SGMVP for research and development is indeed extensive:

1) Analysis and enhanced understanding of power system behaviour: The SGMVP platform provides power system researchers and engineers with convenient access to real-world grid operational and PMU data with sophisticated visualization functions. This provides a valuable tool for comprehensive analysis of power system behaviours for enhanced understanding of the system, e.g. under and over frequency disturbances, inter-area oscillations, etc. This is particularly important with the increasing penetration of renewable energy, where the system behaviour has changed significantly and is expected to continuously change in the future compared with a conventional system dominated by synchronous generation.

2) Power system model validation: When validating the power system model against the real system, it is ideal to compare the model's behaviour with the actual behaviour of the system during the system events to be studied. Presently, one of the key challenges of validating power system models is the difficulty in replicating the system operating conditions when the disturbances occur - either lacking grid operational data or high-resolution frequency, RoCoF and phasor data. As a result, currently the validation is highly dependant on the support from industry partners or publicly available data, which is currently very limited. As discussed in Section II-E, the SGMVP platform constantly monitors the grid status and records any disturbances detected. These records, along with the readily available PMU and grid operational data, provide extensive cases for validating power system models, which strongly supports the simulation work for research and development activities.

3) Source of training data for machine learning applications: Machine learning techniques have been increasingly popular and widely used for addressing power system challenges [23]. Many machine learning techniques, e.g. artificial neural network, require realistic data for training purposes. Currently, the access to comprehensive real-world data is extremely difficult so simulated data is often used for the training purpose, which could compromise the ultimate system performance due to the inherent difference between the model and the real system. The SGMVP can offer an ideal source of training data for machine learning applications in power systems.

4) Data sources for validating wide area monitoring, control and protection systems (WAMPACs): The high-resolution PMU data collected by the SGMVP platform can also be used for testing WAMPACs systems (e.g. for frequency control as reported in [2]). Currently, there are two PMUs available for collecting data, but more PMUs installed at different locations in the GB network are expected to be integrated to the system, which will be an ideal platform for sharing valuable data for researchers and developers of WAMPAC systems. 


\section{B. Education}

There is also a huge potential for the application of the SGMVP platform for facilitating power system engineering education. The sophisticated graphical interface allows intuitive illustration of the grid status, which helps the students to view the real-time system operating condition in an easy and convenient manner. The events log in the platform can also be used as examples for explaining power system disturbances and its behaviour. Furthermore, compared with text-book examples, using real-world data and examples are easier to attract the students' interests and for them to better understand the concepts they learn from the book.

Furthermore, PMU technology is relatively new and has not yet been an established component of power systems teaching. Therefore, the platform can also be an ideal tool for teaching and training courses relating to PMU technologies.

\section{Other applications}

The SGMVP platform can be used for any applications that require the access to or visualization of grid operational data or PMU data. For example, although the energy market participants largely rely on the grid operational data for decision making, the SGMVP offers an alternative to existing platforms with enhanced capability of offering PMU data. This is important for the market participants as a better understanding of the changing dynamics of the system will indicate the need for new solutions for future ancillary services, e.g. faster frequency response as reported in [2]. The SGMVP platform offers an ideal solution for day-to-day energy trading purpose, but also better understanding of the need for future ancillary services, which will have an significant impact on the commercial decisions.

\section{CONCLUSIONS AND FUTURE WORK}

This paper presented the design and implementation of SGMVP, the UK's first open platform for acquiring, archiving and visualizing high-resolution real-time measurement data from PMUs and grid operational data. In the paper, the hardware architecture for supporting the SGMVP platform has been presented, along with the design and implementation of various functional blocks for data acquisition, archiving, monitoring and visualization. The SGMVP platform provides an ideal solution for open and convenient access to valuable grid data, which enables a wide range of potential applications in research and eduction (e.g. model validation, data-driven approaches in power systems, teaching of power system courses, etc.).

Currently, there are two PMUs installed at two locations used for real-time PMU data collection. Since renewable and synchronous generation are unevenly distributed in the network, the GB power system has regional variations in frequency and RoCoF during disturbances. Therefore, future work will focus on incorporating more PMUs in various locations across the network in order to capture a more comprehensive set of measurement data of the entire GB grid.

\section{REFERENCES}

[1] National Grid, "System Operability Framework 2016," Tech. Rep., 2016.

[2] Q. Hong, M. Nedd, S. Norris, I. Abdulhadi, M. Karimi, V. Terzija, B. Marshall, K. Bell, and C. Booth, "Fast frequency response for effective frequency control in power systems with low inertia," The Journal of Engineering, vol. 2019, no. 16, pp. 1696-1702, 2019.

[3] P. Wall, N. Shams, V. Terzija, et al., "Smart frequency control for the future gb power system," in 2016 IEEE PES ISGT-Europe, Oct 2016, pp. 1-6.

[4] D. Wilson, S. Clark, et al., "Advances in Wide Area Monitoring and Control to address Emerging Requirements related to Inertia, Stability and Power Transfer," in CIGRE Paris Session, 2016.

[5] S. Blair, M. Syed, E. Guillo Sansano, Q. Hong, C. Booth, G. Burt, A. Hinojos, and I. Avila, "Review of approaches for using synchrophasor data for real-time wide-area control," 12 2018, first IEEE International Conference on Smart Grid Synchronized Measurements and Analytics.

[6] Grid Watch Templar, "GB National Grid Status," https://gridwatch. templar.co.uk/index.php, [Online; accessed 14-July-2019].

[7] Mark Stolworthy, "Grid Watch," http://gridwatch.co.uk/, [Online; accessed 14-July-2019].

[8] University of Tennessee, "FNET/GridEye Web Display," http:// fnetpublic.utk.edu/index.html, [Online; accessed 14-July-2019].

[9] Swissgrid, "Swissgrid: Current grid key figures," https: //www.swissgrid.ch/en/home/operation/grid-data/current-data.html\# wide-area-monitoring, [Online; accessed 14-July-2019].

[10] "MedFasee," http://www.medfasee.ufsc.br/temporeal/, [Online; accessed 14-July-2019].

[11] University of Strathclyde, "Technology and Innovation Centre," https:// www.strath.ac.uk/research/technologyandinnovationcentre/, [Online; accessed 14-July-2019].

[12] C. Booth, F. Coffele, and G. Burt, "The power networks demonstration centre: An environment for accelerated testing, demonstration and validation of existing and novel protection and automation systems," in 12th IET DPSP 2014, March 2014, pp. 1-6.

[13] Schweitzer Engineering Laboratories, Inc., SEL-451-5 Protection, Automation, and Bay Control system, 2018.

[14] Alstom, Reason RPV311 Technical Manual, 2014.

[15] University of Strathclyde, "Smart Grid Monitoring and Visualisation Platformion Centre," http://smartgridmvp.eee.strath.ac.uk/sgmvp/, [Online; accessed 14-July-2019].

[16] Elexon, "Balancing Mechanism Reporting Service (BMRS)," https: //www.bmreports.com/, [Online; accessed 14-July-2019].

[17] _ "BMRS API and Data Push User Guide," https://www.elexon. co.uk/guidance-note/bmrs-api-data-push-user-guide/, [Online; accessed 14-July-2019].

[18] S. Sandi, B. Krstajic, and T. Popovic, "pypmu - open source python package for synchrophasor data transfer," in 24th Telecommunications Forum (TELFOR), 2016.

[19] "IEEE Std C37.118.1-2011 - IEEE Standard for Synchrophasor Measurements for Power Systems," 2011.

[20] "MySQL," https://www.mysql.com/, [Online; accessed 14-July-2019].

[21] "Advanced Python Scheduler," https://apscheduler.readthedocs.io/, [Online; accessed 14-July-2019].

[22] "Highcharts Demos," https://www.highcharts.com/demo, [Online; accessed 14-July-2019].

[23] E. Hossain, I. Khan, F. Un-Noor, S. S. Sikander, and M. S. H. Sunny, "Application of big data and machine learning in smart grid, and associated security concerns: A review," IEEE Access, vol. 7, pp. 13960 13988,2019 